\title{
Distribuição binomial negativa e multiplicidades em colisões entre prótons
}

\author{
Negative binomial distribution and multiplicities in proton collisions
}

\author{
Paulo César Beggio*10
}

${ }^{1}$ Universidade Estadual do Norte Fluminense Darcy Ribeiro, Laboratório de Ciências Matemáticas, Campos dos Goytacazes, RJ, Brasil.

Recebido em 24 de Maio, 2018. Revisado em 20 de Agosto, 2018. Aceito em 20 de Agosto, 2018.

\begin{abstract}
A produção de partículas em experimentos de colisões entre prótons fornece informações fundamentais dos mecanismos de conversão da energia inicial dos prótons num determinado número de partículas secundárias através da medida de sua Distribuição de Multiplicidade em interações nucleares fortes. Nesse contexto o Modelo Binomial Negativo tem sido extensivamente utilizado em estudos teóricos e em parametrizações das informações experimentais tornando-se, portanto, importante o conhecimento das hipóteses envolvidas na elaboração do modelo, de forma a propiciar adequada aplicação e interpretação de resultados. Efetuamos assim uma discussão do Modelo Binomial Negativo baseando-se na realização de um experimento aleatório clássico, que conduz e auxilia na obtenção da expressão analítica da Função de Probabilidade. Expressamos ainda a referida Função de Probabilidade em termos da Função Gama, da variável multiplicidade e da multiplicidade média adotando um procedimento matemático não encontrado na literatura específica. Implicações da aplicação do modelo no estudo de Distribuições de Multiplicidades em colisões entre prótons são discutidas.
\end{abstract}

Palavras-chave: Distribuições de Multiplicidades; Distribuição Binomial Negativa; Produção Múltipla de Partículas; Colisões de Prótons.

Particle production in proton collision experiments provides fundamental information on the mechanisms of initial energy conversion of protons in a number of secondary particles by measuring their Multiplicity Distribution in strong nuclear interactions. In this context, the Negative Binomial Model has been extensively used in theoretical studies and parameterization of experimental information, making it important to know the hypotheses involved in the elaboration of the model, in order to provide adequate application and interpretation of results. We thus conduct a discussion of the Negative Binomial Model based on the realization of a classic random experiment, which leads and assists in obtaining the analytical expression of the Probability Function. We also express the said Function of Probability in terms of the Gamma Function, the multiplicity variable and the average multiplicity adopting a mathematical procedure not found in the specific literature. Implications of the application of the model in the study of Multiplicities Distributions in collisions between protons are discussed.

Keywords: Multiplicity distributions; Negative Binomial distritibution; Multiparticle production; Proton collisions.

\section{Introdução}

Prótons são partículas que constituem o núcleo atômico e possuem complexa estrutura interna composta de outras partículas denominadas quarks e glúons, que são algumas das partículas elementares que constituem a matéria 1 , 2 - Tendo como um dos propósitos compreender a estrutura da matéria e suas interações, prótons são acelerados em feixes e colididos em energias de colisão da ordem de $10^{9}-10^{12}$ elétron-Volts [2] . Nessa ordem de energia de colisão quarks e glúons interagem predominantemente através da força nuclear forte [1, 2] e um dos resultados das colisões entre prótons, e das consequentes interações entre quarks e glúons, é a criação de um conjunto de novas partículas, processo esse denominado produção múltipla

*Endereço de correspondência: pcbeggio@gmail.com de partículas 3 . Em geral, denotamos o número de partículas produzidas por " $N$ " $(N=2,4,6, \ldots n \ldots)$ e " $P(N)$ "denota sua probabilidade de produção. Neste artigo discutimos o conjunto $\{N, P(N)\}$, denominado Distribuição de Multiplicidade (DM) das partículas produzidas nas colisões entre prótons, o qual constitui-se numa quantidade física experimental fundamental para o entendimento dos mecanismos de interações nucleares fortes de quarks e glúons [3. Dados experimentais sobre DM foram disponibilizados através dos experimentos CERN ISR (1984), CERN UA5 Collaboration (1987-1989) e do Experimento E735 realizado no Tevatron (1998) 4-7. Atualmente, o Grande Colisor de Hádrons, ou LHC (Large Hadron Collider), que é o maior acelerador de partículas de toda história [2], tem disponibilizado novas informações experimentais sobre DM. Detalhes sobre os experimentos 
no LHC podem ser encontrados nas Refs. [2,8]. Objetivando então compreender os mecanismos de produção de partículas e havendo dados experimentais sobre DM em amplo intervalo de energia de colisão, são necessários modelos matemáticos e/ou Distribuições de Probabilidades para serem efetuados os estudos e investigações 9 16]. Nesse contexto a Distribuição de Probabilidade Binomial Negativa (DBN), também referida como Distribuição de Pascal, tem sido extensivamente utilizada para estudar e parametrizar DM's observadas nos experimentos de colisões entre prótons e próton-antipróton 15 17]. A ampla utilização do Modelo Binomial Negativo em diversas análises motivou o desenvolvimento deste trabalho e que tem dois propósitos centrais. Um deles é apresentar uma discussão didática do Modelo Binomial Negativo e, outro, incentivar novas pesquisas sobre multiplicidades devido à relevância do tema para o entendimento da dinâmica de produção de partículas. Assim, devido a proposta didática do trabalho, na próxima seção apresentamos conceitos necessários e direcionados para definir Distribuição de Probabilidade, revendo a Distribuição de Bernoulli com o propósito de definir a notação a ser utilizada e discutindo também aspectos sobre independência de eventos. Na Seção 3 apresentamos e discutimos nossa proposta para obtenção da DBN, explorando um experimento aleatório clássico. Expressamos também a fórmula analítica da DBN em termos da Função Gama e da variável " $n$ ", como frequentemente utilizada nas aplicações para descrever Distribuições de Multiplicidades. $\mathrm{Na}$ Seção 4 são apresentadas as considerações finais.

\section{Sobre a definição de distribuição de probabilidade}

Admitimos que os leitores tenham conhecimentos sobre conceitos de probabilidades tais como experimento aleatório, eventos, espaço amostral e definição clássica para cálculo de probabilidades. No trabalho com probabilidades definimos os eventos e então calculamos a probabilidade de ocorrência desses eventos usando a definição clássica para o cálculo de probabilidades 18,19]. Baseado em exemplo discutido na Ref. [18, considere o experimento aleatório: "lançamento de duas moedas honestas", o qual tem como espaço amostral o conjunto $\{c c, c r, r c, r r\}$, onde " $c$ " representa cara e " $r$ " coroa. Calculando as probabilidades dos eventos:

$A=$ Ocorrência de nenhuma cara;

$B=$ Ocorrência de uma cara;

$C=$ Ocorrência de duas caras;

obtemos:

$$
P(A)=\frac{1}{4}, P(B)=\frac{1}{2}, P(C)=\frac{1}{4} .
$$

Um procedimento matemático formal pode ser adotado para o cálculo dessas probabilidades. Procedimento esse que consiste em representar os eventos por números ao invés de palavras. Isso pode ser feito introduzindo o conceito de Variável Aleatória (VA) X e definida, nesse exemplo específico, como:

$$
X \text { : Número de caras. }
$$

Como estamos agora interessados na representação dos eventos através de números, e não mais por palavras, observamos então que $X$ pode assumir os valores $X=$ $\{0,1,2\}$ para representar os eventos $A, B$ e $C$, e com as seguintes correspondências:

$X=0 \rightarrow$ Ocorrência de nenhuma cara (Evento $A$ );

$X=1 \rightarrow$ Ocorrência de uma cara (Evento $B$ );

$X=2 \rightarrow$ Ocorrência de duas caras (Evento $C$ ).

Em notação geral escrevemos:

$$
X=\left\{x_{1}, x_{2}, \ldots, x, \ldots\right\}
$$

para indicar os possíveis valores da VA $X$. Esquematicamente:

Tabela 1: Valores possíveis para a variável aleatória $X$, definida em (2), e respectivos valores das probabilidades.

\begin{tabular}{|c|c|c|}
\hline$X$ & $P(X)$ & Evento correspondente \\
\hline 0 & $1 / 4$ & Nenhuma cara $(A)$ \\
\hline 1 & $1 / 2$ & Uma cara $(B)$ \\
\hline 2 & $1 / 4$ & Duas caras $(B)$ \\
\hline
\end{tabular}

Nas duas colunas numéricas da Tabela 1 identificamos o conjunto $\{X, P(X)\}$, o qual é denominado Distribuição de Probabilidade da VA $X$ [20], de fácil representação gráfica. Observamos que o tratamento sobre VA é naturalmente mais abrangente [18,20]. Efetuamos aqui uma discussão direcionada para introduzir o conceito de Função de Probabilidade.

\subsection{Distribuição de probabilidade - Modelo de Bernoulli}

A repetição de sucessivos ensaios de Bernoulli é fonte de vários problemas teóricos interessantes [21], originando outros modelos tais como o Binomial, Geométrico e mesmo o Binomial Negativo. Assim, abordamos alguns aspectos relevantes que conduzem à obtenção da expressão analítica da Função Distribuição de Probabilidade nesse modelo e também para definir a notação a ser utilizada. Todo experimento aleatório no qual distinguimos apenas dois resultados possíveis, e mutuamente exclusivos, é denominado experimento ou ensaio de Bernoulli [20]. Eventos mutuamente exclusivos são eventos que não podem ocorrer conjuntamente, $A \cap B=\emptyset$. Um exemplo é a realização do experimento aleatório "lançamento de uma moeda", naturalmente, só há dois resultados possíveis e que são mutuamente exclusivos, ou seja, a ocorrência de cara exclui a possibilidade de ocorrência de coroa e vice-versa. Considere agora uma única realização de qualquer experimento classificável como experimento de Bernoulli e no qual definimos, por convenção, um dos resultados como sucesso $(S)$ e o outro como fracasso $(F)$. Indicamos o valor da probabilidade de ocorrer sucesso 
pelo algarismo $p$ e o valor da probabilidade de fracasso por $q$. Por serem eventos mutuamente exclusivos resulta que $p+q=1$. Como exemplo recorremos ao experimento referido, "lançamento de uma moeda", e definimos a ocorrência de cara como sucesso (com probabilidade $p=1 / 2$ ) e a ocorrência de coroa como fracasso $(q=1 / 2)$. Note que a definição de sucesso é arbitrária. Como mencionado, a VA deve expressar numericamente os resultados possíveis e pode ser definida no modelo de Bernoulli 18 como:

\section{$X$ : Número de sucessos em única realização de um experimento de Bernoulli.}

Sendo que o experimento é realizado uma única vez, a VA $X$ assume os valores iguais a 1 e 0 , onde $X=1$ indica a ocorrência do resultado sucesso e $X=0$ indica a ocorrência de fracasso. Assim $X=\{0,1\}$ e podemos escrever: $P(S)=P(X=1)=p$ e $P(F)=P(X=0)=q$, na forma tabular:

Tabela 2: Representação tabular dos resultados da realização de um experimento de Bernoulli.

\begin{tabular}{|c|c|}
\hline$X$ & $P(X)$ \\
\hline 0 & $q$ \\
\hline 1 & $p$ \\
\hline
\end{tabular}

A expressão analítica que representa a Função de Probabilidade no modelo de Bernoulli é então escrita na forma:

$$
P(X=x)=p^{x} q^{1-x},
$$

produzindo, naturalmente, os valores indicados na Tabela 2 Notamos que a Função de Probabilidade é definida como a função que atribui a cada valor assumido pela VA a probabilidade do evento correspondente 18.

\subsection{Eventos independentes e multiplicação de probabilidades}

A multiplicação dos valores das probabilidades, $p$ e $q$, que decorrem da independência entre as várias realizações do mesmo experimento aleatório, é condição necessária para a dedução da expressão analítica da Função Distribuição de Probabilidade no Modelo Binomial Negativo, a partir de uma generalização do Modelo de Bernoulli. Em geral, discussões envolvendo independência entre eventos são abordadas juntamente com discussões sobre probabilidade condicional [18, 19]. Assim, considere dois eventos $A$ e $B$ e que pertencem ao mesmo espaço amostral. Define-se probabilidade condicional de ocorrer o evento $A$ sabendo-se que ocorreu o evento $B$, denotada por $P(A / B)$, na forma 19 :

$$
P(A / B)=\frac{P(A \cap B)}{P(B)},
$$

onde $P(A \cap B)$ denota a probabilidade de intersecção entre os eventos $A$ e $B$ [19], ou seja, denota a probabilidade de ocorrer o evento $A$ e o evento $B . P(A)$ e $P(B)$ denotam probabilidades de ocorrência dos eventos $A$ e $B$, tal que $P(B) \neq 0$ em (6).

Como exemplo de aplicação da probabilidade condicional considere o experimento aleatório "lançamento de um dado", onde definimos os eventos:

$A=$ Sair o número 4; e

$B=$ Sair número par.

O cálculo da probabilidade condicional resulta $P(A / B)=$ $\frac{1}{3}$, significando a probabilidade de sair o número 4 sabendo que ocorreu número par no lançamento do dado. Notamos agora que importante consequência da definição de probabilidade condicional é obtida ao escrevermos a expressão (6) na forma [19]:

$$
P(A \cap B)=P(A / B) \cdot P(B) .
$$

A expressão (7) é também referida como "Teorema da Multiplicação de Probabilidades" [19]. Referente à independência, dois eventos $A$ e $B$ são independentes se a informação de ocorrência de $B$ não altera a probabilidade atribuída ao evento $A$ [19,20], assim escrevemos:

$$
P(A / B)=P(A) \text {. }
$$

Substituindo (8) em (7) define-se [18, 19] formalmente que os eventos A e B são independentes se, e somente se,

$$
P(A \cap B)=P(A) \cdot P(B) .
$$

A última expressão significa que a probabilidade de ocorrer um evento $A$ e um evento $B$, sendo eles independentes, é calculada pelo produto das probabilidades de ocorrência desses eventos. A Eq. (9) é generalizada para o caso em que temos a ocorrência de vários eventos independentes. Portanto, se os eventos $A_{1}, A_{2}, \ldots \ldots, A_{m}$, são independentes [18] então:

$$
P\left(\cap_{i=1}^{m} A_{i}\right)=P\left(A_{1}\right) \cdot P\left(A_{2}\right) \ldots \ldots P\left(A_{m}\right) .
$$

É oportuno notar que se dois eventos $A$ e $B$ são mutuamente exclusivos, então eles são eventos dependentes, pois se $A$ ocorre o evento $B$ não ocorre. Ou seja, a ocorrência de um evento condiciona a não ocorrência do outro evento [18.

\section{Função de probabilidade - Modelo Binomial Negativo e Aplicação}

Obtemos nesta Seção a Função de Probabilidade no modelo Binomial Negativo subsidiados pelos elementos apresentados na última Seção. Recorremos à analogia com a realização de um experimento aleatório simples para exemplificar algumas etapas da elaboração do modelo.

\subsection{Lançamentos de uma moeda e a Função de Probabilidade}

Considere várias repetições do experimento "lançamento de uma moeda honesta". Como discutido em 2.1, cada 
lançamento é um experimento de Bernoulli havendo, naturalmente, dois resultados possíveis e mutuamente exclusivos. Na Seção anterior definimos sucesso como a ocorrência de cara com valor de probabilidade $p$, a qual permanece constante em cada repetição do experimento e fracasso a ocorrência de coroa com probabilidade $q$. Considere agora que dentre as várias possíveis repetições do experimento desejamos obter dois sucessos e adotamos a letra $k$ para essa indicação. Assim $k=2$ significa que queremos obter 2 sucessos ( 2 caras) em várias repetições do experimento aleatório "lançamento de uma moeda honesta". Se atribuirmos à VA $X$ o significado: "número de vezes que devemos repetir o experimento até se obter 2 sucessos", temos que $X$ poderá assumir os valores numéricos:

$$
X=\{2,3,4,5, \ldots \ldots\} .
$$

Naturalmente, se queremos obter $k=2$ sucessos o experimento terá que ser repetido no mínimo 2 vezes, implicando assim que o primeiro valor possível para a VA será o número de sucessos desejados, como indicado em (11). A situação exemplificada é generalizada no contexto do Modelo Binomial Negativo, onde a VA é formalmente enunciada como 18, 19]:

\section{$X$ : Número de repetições de um experimento de}

Bernoulli até se obter um número $k$ de sucessos.

Então $X$ poderá assumir os valores:

$$
X=\{k, k+1, k+2, k+3, \ldots \ldots\} .
$$

Ressaltamos que a definição da VA, em (12), tem como implicação que o resultado da última repetição do experimento será sempre sucesso, visto que devemos repetir o experimento até obter o número $k$ de sucessos pretendidos. Para orientar a obtenção da Função de Probabilidade construímos a representação indicada na Tabela 3 reportando-nos novamente ao experimento aleatório "lançamento de uma moeda". Também fixamos a ocorrência de dois sucessos, $k=2$, ou seja, duas caras e que são representadas na Tabela 3 por sua probabilidade de sucesso $p$. Na primeira linha horizontal indicamos os números de repetições, considerando até cinco repetições do experimento e sendo essa uma escolha arbitrária. Na primeira coluna à esquerda indicamos alguns valores possíveis da VA e que representam o número total de repetições do experimento aleatório. Na última coluna à direita são indicadas as proporcionalidades entre a Função de Probabilidade, $P(X=x)$, e os valores das probabilidades de sucesso, $p$, e de fracasso $q$. Como devemos efetuar sucessivas repetições do experimento até obtermos os dois sucessos pretendidos $(k=2)$, apresentamos a seguinte interpretação da Tabela 3 .

$x=2$ : tem como significado obter $k=2$ sucessos, em $x=2$ repetições do experimento aleatório, sendo $p$ a probabilidade constante em cada repetição.

$x=3$ : significa obter $k=2$ sucessos em $x=3$ repetições do experimento, e assim sucessivamente.

Note que a partir deste ponto temos combinações de resultados e usamos negrito para ressaltar os sucessos nas combinações possíveis. Especificamente, para obtermos $k=2$ sucessos em $x=3$ repetições do experimento, há duas combinações de resultados possíveis e indicadas nas $5^{\mathrm{a}}$ e $6^{\mathrm{a}}$ linhas. São elas sucesso, fracasso, sucesso e representada por $(\boldsymbol{p} q \boldsymbol{p})$ na notação adotada. A outra possibilidade é fracasso, sucesso, sucesso ou simplesmente $(q \boldsymbol{p} \boldsymbol{p})$. Como mencionado, devido à definição da VA nesse modelo, o último lançamento resulta sempre em sucesso, pois interrompemos as repetições do experimento quando o número $k$ de sucessos é atingido. Continuando a interpretação da Tabela 3 notamos que para obtermos $k=2$ sucessos em $x=4$ repetições do experimento, há 3 combinações de resultados possíveis e indicadas na $8^{\mathrm{a}}, 9^{\mathrm{a}}$

Tabela 3: Representação possível para orientar a obtenção da Função de Probabilidade no Modelo Binomial Negativo considerando o experimento aleatório "lançamento de uma moeda honesta". A ocorrência de sucesso numa repetição do experimento é representada por $p$ e a de fracasso por $q$. As repetições do experimento aleatório e seus resultados são independentes entre si, implicando na multiplicação das probabilidades $p$ e $q$. Usamos negrito para destacar o deslocamento da probabilidade associada ao evento sucesso nas combinações possíveis. Na última coluna à direita são indicadas a proporcionalidade entre a Função de Probabilidade e as probabilidades $p$ e $q$.

\begin{tabular}{|l|l|l|l|l|l|l|}
\hline & $1^{\mathrm{O}}$ & $2^{\mathrm{o}}$ & $3^{\mathrm{O}}$ & $4^{\mathrm{O}}$ & $5^{\mathrm{O}}$ & \\
\hline & & & & & & $\begin{array}{l}\text { Proporcionalidades entre Função de Probabili- } \\
\text { dade } P(X=x) \text { e as probabilidades } p \text { e } q .\end{array}$ \\
\hline & $p$ & $p$ & & & & $P(X=2) \alpha p p$ ou $P(X=2) \alpha p^{2}$ \\
\hline$x=3$ & $p$ & $q$ & $p$ & & & $P(X=3) \alpha q p^{2}$ \\
\hline & $q$ & $p$ & $p$ & & & $P(X=3) \alpha q p^{2}$ \\
\hline$x=4$ & $p$ & $q$ & $q$ & $p$ & & $P(X=4) \alpha q^{2} p^{2}$ \\
\hline & $q$ & $p$ & $q$ & $p$ & & $P(X=4) \alpha q^{2} p^{2}$ \\
\hline & $q$ & $q$ & $p$ & $p$ & & $P(X=4) \alpha q^{2} p^{2}$ \\
\hline$x=5$ & $p$ & $q$ & $q$ & $q$ & $p$ & $P(X=5) \alpha q^{3} p^{2}$ \\
\hline & $q$ & $p$ & $q$ & $q$ & $p$ & $P(X=5) \alpha q^{3} p^{2}$ \\
\hline & $q$ & $q$ & $p$ & $q$ & $p$ & $P(X=5) \alpha q^{3} p^{2}$ \\
\hline & $q$ & $q$ & $q$ & $p$ & $p$ & $P(X=5) \alpha q^{3} p^{2}$ \\
\hline
\end{tabular}


e $10^{\mathrm{a}}$ linhas. São elas sucesso, fracasso, fracasso, sucesso $(\boldsymbol{p} q q \boldsymbol{p})$, fracasso, sucesso, fracasso, sucesso $(q \boldsymbol{p} q \boldsymbol{p})$, e fracasso, fracasso, sucesso, sucesso ( $q q \boldsymbol{p} \boldsymbol{p})$. As combinações possíveis para obtermos $k=2$ sucessos em $x=5$ repetições do experimento são indicadas da $12^{\mathrm{a}}$ a $15^{\mathrm{a}}$ linhas. É fundamental observarmos que as várias repetições do experimento e seus respectivos resultados, de sucesso ou fracasso, são independentes uns dos outros e implicando que devemos efetuar o produto das probabilidades de sucesso e fracasso para obter a expressão analítica da Função de Probabilidade. Especificamente, a realização e o resultado do $1^{\mathrm{O}}$ lançamento da moeda não interfere no resultado do $2^{\mathrm{O}}$ lançamento. Por sua vez, os resultados do $1^{\mathrm{o}}$ e $2^{\mathrm{o}}$ lançamentos não interferem no resultado do $3^{\circ}$ lançamento, implicando que as várias repetições do experimento e seus respectivos resultados são independentes entre si, como discutido na Subseção 2.2. Assim, para obtermos a expressão analítica da Função de Probabilidade, recorremos à Eq. (10) e efetuamos o produto dos valores das probabilidades, $p$ ou $q$, associadas aos resultados de sucesso ou fracasso obtidos em cada repetição do experimento. Baseado na Tabela 3 verificam-se as proporcionalidades entre a Função de Probabilidade $P(X=x)$ e os valores das probabilidades $p$ e $q$, a saber: $x=2 \Rightarrow P(X=2) \alpha p^{2}$, significando $k=2$ sucessos em $x=2$ repetições do experimento aleatório.

$x=3 \Rightarrow P(X=3) \alpha p q p$, significando $k=2$ sucessos em $x=3$ repetições independentes do experimento. Esse caso implica que um dos resultados é fracasso, com probabilidade $q$. Devemos então considerar também a outra configuração possível, ou seja,

$$
P(X=3) \alpha q p p, \text { ou } P(X=3) \alpha q p^{2} .
$$

Importante observar, nesse caso, que a probabilidade de obter $k=2$ sucessos em $x=3$ repetições do experimento é calculada por:

$$
P(X=3)=2 q p^{2},
$$

onde o fator 2 expressa o número das duas configurações possíveis. Prosseguindo em nossa interpretação, para $x=4$ existem as configurações:

$$
\begin{aligned}
& P(X=4) \alpha p q q p \Rightarrow P(X=4) \alpha q^{2} p^{2}, \\
& P(X=4) \alpha q p q p \Rightarrow P(X=4) \alpha q^{2} p^{2}, \\
& P(X=4) \alpha q q p p \Rightarrow P(X=4) \alpha q^{2} p^{2} .
\end{aligned}
$$

Como há 3 configurações possíveis, referentes à obtenção de $k=2$ sucessos em $x=4$ repetições do experimento, escrevemos:

$$
P(X=4)=3 q^{4-2} p^{2} .
$$

Ressaltamos que o fator 3 expressa o número de configurações possíveis nesse caso. Antes de prosseguirmos é conveniente expressar a Função de Probabilidade em termos das variáveis $x$ e $k$. Assim a Eq. (15) é, ainda parcialmente, reescrita na forma:

$$
P(X=x)=3 q^{x-k} p^{k} .
$$

Nas Eqs. (14) e (15) os fatores 2 e 3 representam respectivamente os números de configurações possíveis e são agrupamentos denominados Combinações [19]. Exemplificamos o cálculo do número das combinações possíveis nesse modelo recorrendo novamente ao exemplo da Tabela 3 , de obter $k=2$ sucessos em $x=4$ repetições do experimento. Como o resultado da última repetição é sempre sucesso temos que o outro sucesso $(2-1)=1$, ou $(k-1)$, poderá ocorrer em qualquer uma das outras $(4-1)=3$, ou $(x-1)$, repetições do experimento. $\mathrm{O}$ fator 1 representa a exclusão do sucesso que ocorre no último lançamento. Em outras palavras, desde que a última repetição resulta sempre em sucesso, o outro sucesso poderá ocorrer no $1^{\mathrm{O}}, 2^{\mathrm{O}}$ ou $3^{\mathrm{O}}$ lançamentos. Sendo assim, o número de combinações possíveis é calculado por 18, 19:

$$
\left(\begin{array}{l}
4-1 \\
2-1
\end{array}\right)=\frac{3 !}{1 ! 2 !}=3
$$

De forma geral:

$$
\left(\begin{array}{l}
x-1 \\
k-1
\end{array}\right)=\frac{(x-1) !}{(k-1) !(x-k) !} .
$$

Assim, em razão das Eqs. (16), (17) e (18), a expressão analítica da Função de Probabilidade, Eq. (16), é escrita na forma:

$$
P(X=x)=\left(\begin{array}{c}
x-1 \\
k-1
\end{array}\right) q^{x-k} p^{k}
$$

ou ainda:

$$
P(X=x)=\left(\begin{array}{l}
x-1 \\
k-1
\end{array}\right)(1-p)^{x-k} p^{k} .
$$

$x \geq k$. A Eq. (20) expressa o fato que a Função de Probabilidade, $P(X=x)$, é proporcional à probabilidade de sucesso $p$ elevada ao número de sucessos desejados, $k$, e proporcional a probabilidade de fracasso $q$ elevada ao número de fracassos e que é calculado por $(x-k)$. A Eq. (20) permite então o cálculo da probabilidade de obtermos $k$ sucessos em $x$ repetições de qualquer experimento classificável como experimento de Bernoulli, sendo $p$ a probabilidade constante em cada repetição do experimento de ocorrer o evento definido como sucesso. Como uma interpretação alternativa podemos afirmar que a Eq. (20) fornece a probabilidade de que antes da ocorrência do número $k$ de sucessos pretendidos, os outros $(k-1)$ sucessos podem estar alocados em qualquer ordem nas $(x-1)$ posições restantes, que representam os resultados das outras repetições do experimento aleatório. Os parâmetros $k$ e $p$ caracterizam essa distribuição, que pode ser representada pela notação $X: B N(k, p)$, significando que a VA $X$ segue a Distribuição de Probabilidade Binomial Negativa e depende dos parâmetros $k$ e $p$.

\subsection{Distribuição de multiplicidade}

Com a finalidade de propiciar a conexão entre os conceitos sobre a DBN e a quantidade física Distribuição de Multiplicidade, tecemos alguns comentários no sentido de 
ilustrar de forma simples e qualitativa essa quantidade física. Como mencionado, prótons são partículas com complexa estrutura interna composta de quarks e glúons. A Distribuição de Multiplicidade é sensível ao número de colisões entre quarks e glúons contidos nos prótons colidentes e, em geral, aos mecanismos fundamentais de produção de partículas 22]. Especificamente, nas colisões entre dois prótons pode haver a criação de 2 partículas, ou de 4 , ou de 6 ou, de forma geral, pode haver a produção de " $n$ " partículas. Notamos que " $n$ " é um número par devido à conservação da carga elétrica no processo de produção de novas partículas [1]. Para abordagem do problema definimos a VA:

\section{$N$ : Número de partículas produzidas na colisão.}

Tal que:

$$
N=\{2,4,6, \ldots, n, \ldots\}
$$

Esse conjunto é denominado "Conjunto de Multiplicidade das partículas eletricamente carregadas", ou simplesmente "Multiplicidade". A cada elemento " $n$ " é associada sua correspondente probabilidade de produção $P(N=n)$, ou seja, ao valor da multiplicidade $n=2$ corresponde o valor da probabilidade $P(2)$, significando a probabilidade que na colisão entre prótons sejam produzidas duas partículas. De forma geral, $P(N=n)$ é o valor da probabilidade que na colisão sejam produzidas " $n$ " partículas. Dessa forma constitui-se o conjunto "Distribuição de Multiplicidade" $\{N, P(N)\}$, que é uma das características mais básicas das colisões de prótons a altas energias e que tem sido objeto de estudos experimentais e teóricos de forma a possibilitar a compreensão da interação nuclear forte. Com propósito apenas pedagógico apresentamos, na Fig. 1. uma ilustração não realista do processo de produção de partículas em colisão entre prótons.

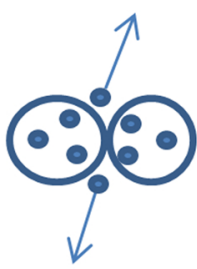

$\{2, P(2)\}$

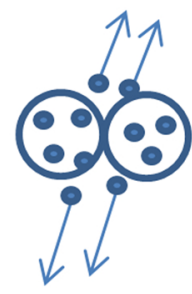

$\{4, P(4)\}$
Figura 1: Ilustração da produção de partículas na colisão entre dois prótons, representados pelos círculos maiores. Círculos menores, no interior dos maiores, representam os constituintes dos prótons. As partículas produzidas na colisão são ilustradas pelos pequenos círculos com setas.

\subsection{Binomial negativa aplicada ao estudo da distribuição de multiplicidade}

A utilização da DBN [10], da superposição de duas DBN [11,23 ou de modelos matemáticos de produção de partículas que utilizam a DBN ou casos limites dela como um elemento do modelo $[9,24,26$ tem fornecido adequadas parametrizações das Distribuições de Multiplicidades. Assim, efetuamos procedimentos matemáticos necessários à aplicação da DBN em análises envolvendo DM, na qual a Função de Probabilidade Binomial Negativa é usualmente expressa em termos da Função Gama e também eliminando-se a variável $x$ e o valor da probabilidade $p$ da expressão (19). Em nosso tratamento vimos que $(x-k)$, na Eq. $(20)$, representa o número de fracassos em $x$ repetições do experimento no qual esperamos obter $k$ sucessos. Introduzimos a variável " $n$ " escrevendo:

$$
n=x-k \Rightarrow x=n+k .
$$

Substituindo (23) em (19) resulta que:

$$
P_{k, p}(n)=\left(\begin{array}{c}
n+k-1 \\
k-1
\end{array}\right) q^{n} p^{k}
$$

Para facilitar o uso da relação complementar [20] seguinte:

$$
\left(\begin{array}{c}
n+l \\
l
\end{array}\right)=\left(\begin{array}{c}
n+l \\
n
\end{array}\right)
$$

efetuamos a mudança de variável: $l=k-1$ na expressão (24) obtendo

$$
P_{k, p}(n)=\left(\begin{array}{c}
n+l \\
l
\end{array}\right) q^{n} p^{k}
$$

Usando então a relação (25) e notando que $p+q=1$, a Eq. (26) é reescrita na forma:

$$
P_{k, p}(n)=\left(\begin{array}{c}
n+k-1 \\
n
\end{array}\right)(1-p)^{n} p^{k},
$$

ou equivalentemente

$$
P_{k, p}(n)=\frac{(n+k-1) !}{n !(k-1) !}(1-p)^{n} p^{k} .
$$

Devido à introdução da variável " $n$ "e seu significado, Eq. (23), a expressão (28) fornece a probabilidade de ocorrer $n$ fracassos e $(k-1)$ sucessos em qualquer ordem, antes de ocorrer um número $k$ de sucessos num experimento de Bernoulli com probabilidade de sucesso $p$ 3. Cálculos envolvendo fatoriais são, em geral, algebricamente trabalhosos tornando-se então conveniente expressar a Eq. (28) em termos da Função Gama e definida 27 como:

$$
\Gamma(n)=\int_{0}^{\infty} e^{-x} x^{n-1} d x
$$

que converge se $n>0$. Se $n$ for um número inteiro positivo, recorremos à identidade [27]:

$$
n !=\Gamma(n+1) \text {. }
$$


Assim, os fatoriais presentes na Eq. (28) são expressos em termos da Função Gama, a saber:

$$
\begin{gathered}
(k-1) !=\Gamma(k) . \\
(n+k-1) !=\Gamma(n+k) .
\end{gathered}
$$

Substituindo (30), (31) e (32) na Eq. (28) resulta:

$$
P_{k, p}(n)=\frac{\Gamma(n+k)}{\Gamma(n+1) \Gamma(k)}(1-p)^{n} p^{k}
$$

Decorre ainda que em aplicações práticas, com frequência, a probabilidade $p$ não é conhecida, entretanto o valor médio da uma amostra de dados experimentais pode ser obtido 28. Assim, sendo $\langle n\rangle$ a multiplicidade média do conjunto $N$ de partículas produzidas nas colisões entre prótons, a mesma é relacionada à probabilidade $p$ de sucesso pela equação [3]:

$$
p^{-1}=1+\frac{<n>}{k} \Rightarrow p=\frac{k}{k+<n>} .
$$

Substituindo (34) em (33) resulta que:

$$
\begin{aligned}
P(n, k,<n>) & =\frac{\Gamma(n+k)}{\Gamma(n+1) \Gamma(k)}\left[1-\frac{k}{<n>+k}\right]^{n} \\
& \times\left[\frac{k}{<n>+k}\right]^{k}
\end{aligned}
$$

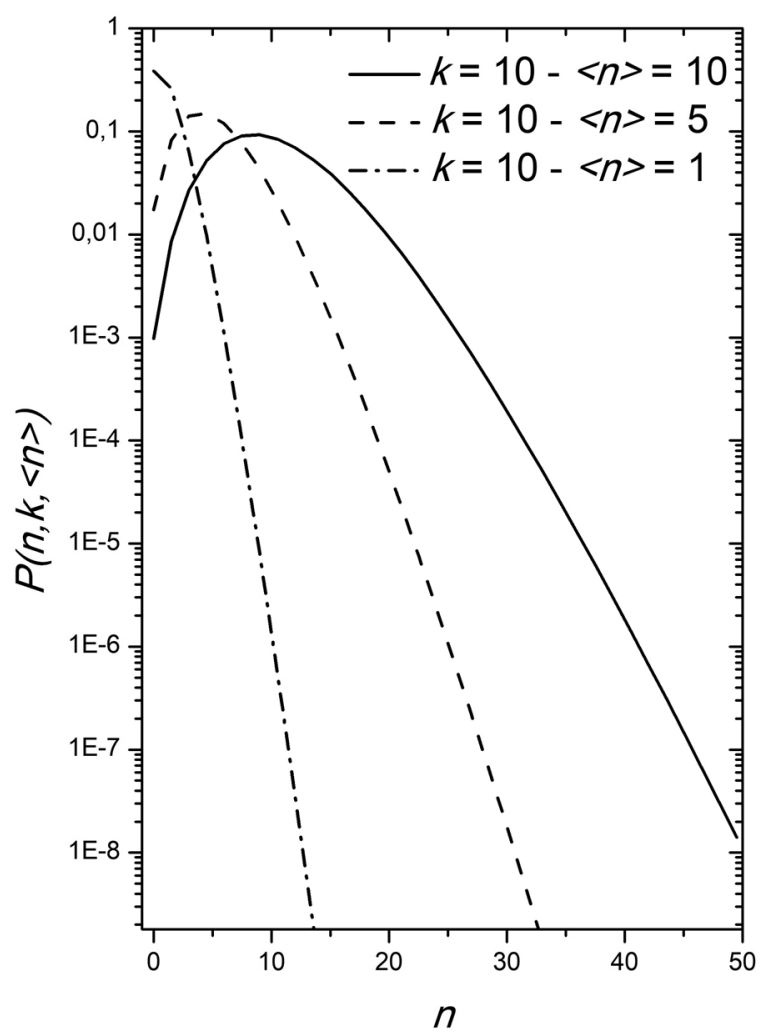

Após efetuarmos algum trabalho algébrico na Eq. (35) obtemos a Função de Probabilidade na forma frequentemente utilizada em investigações de multiplicidades $[3]$ :

$$
\begin{aligned}
P(n, k,<n>) & =\frac{\Gamma(n+k)}{\Gamma(n+1) \Gamma(k)}\left[\frac{<n>/ k}{1+<n>/ k}\right]^{n} \\
& \times \frac{1}{[1+<n>/ k]^{k}} .
\end{aligned}
$$

A forma analítica da DBN depende agora dos valores dos parâmetros $\langle n\rangle$ e $k$, que podem ser determinados em ajustes da Eq. (36) aos dados experimentais correspondentes. A expressão (36) permite também versátil elaboração de gráficos e consequentes comparações com os dados experimentais, se comparada com a Eq. (28). Valendo-se da Eq. (36) apresentamos na Figura 2 gráficos da DBN. No painel esquerdo da figura o valor de $k$ é fixado, enquanto os valores médios, $\langle n\rangle$, são variados. Nota-se que a medida que $\langle n\rangle$ diminui ocorre o estreitamento da distribuição. No painel da direita $\langle n\rangle$ é mantido constante e a medida que $k$ diminui verifica-se a abertura da distribuição.

\section{Discussão e considerações finais}

Desde a primeira aplicação da DBN pela Colaboração UA5 em 1985 [29], a mesma tem sido frequente e amplamente utilizada em investigações envolvendo Distri-

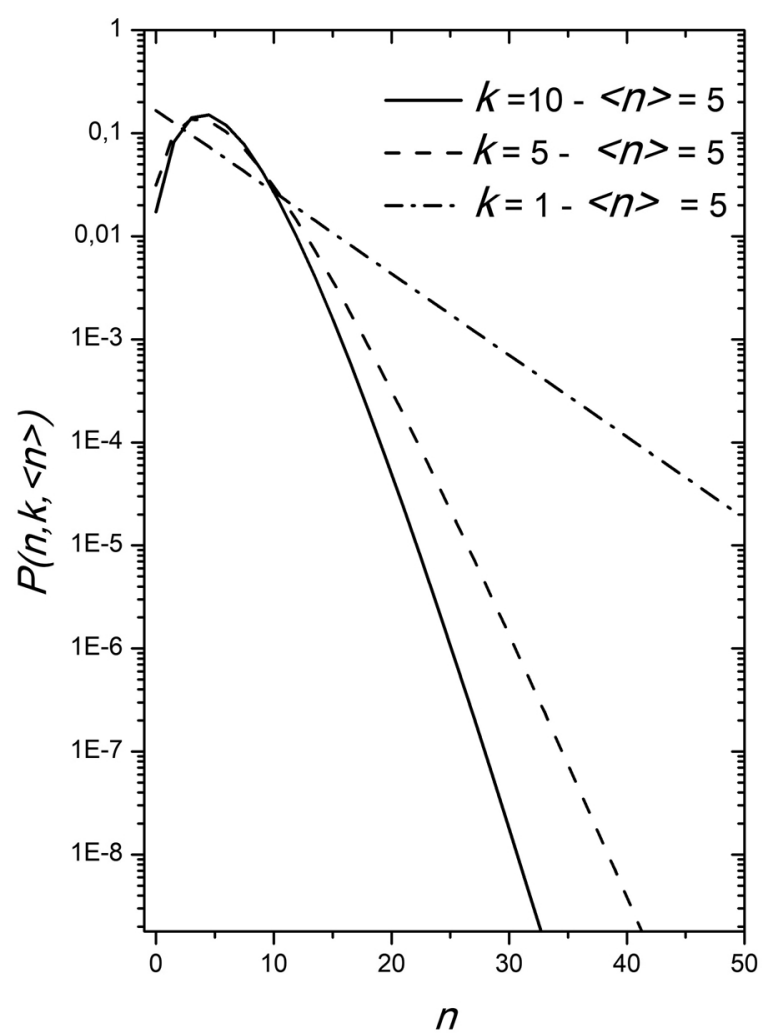

Figura 2: Gráficos da Distribuição Binomial Negativa, Eq. (36). No painel esquerdo o valor do parâmetro $k$ é mantido constante, variando-se os valores do valor médio $\langle n\rangle$. No painel da direita $\langle n\rangle$ é constante e os valores de $k$ são alterados. 
buições de Multiplicidades em sistemas colidentes como próton-próton, próton-antipróton, elétron-pósitron e múonpróton [3]. O fato que resultados experimentais de DM em muitos e diferentes experimentos e em amplo intervalo de energia de colisão podem ser parametrizados pela DBN, pela superposição de duas delas ou mesmo casos limites dessa distribuição não tem sido considerado como um fato acidental e, como apontado por Giovannini e Ugoccioni [11], a impressão é que pode haver uma aproximada regularidade universal nesse fato. Assim, devido ao início das atividades do $\mathbf{L H C}$, disponibilizando novas informações experimentais sobre colisões entre prótons, dispomos de um momento propício para serem efetuados testes, aprimoramentos e desenvolvimentos de modelos matemáticos e/ou estruturas de cálculos para investigações dos mecanismos de produção múltipla de partículas nessas colisões. Com essa motivação discutimos o Modelo Binomial Negativo obtendo sua Função de Probabilidade valendo-se de um cenário simples, propondo uma abordagem didática dos elementos e hipóteses que caracterizam essa distribuição. A finalidade é facilitar e propiciar adequada aplicação e interpretação de resultados, bem como divulgar o tema de pesquisa. Desde que estamos abordando interações entre prótons, é natural esperar que os mecanismos de interações entre quarks e glúons e que produzem partículas, possam ser compreendidos em termos análogos aos elementos que compõem o cenário do Modelo Binomial Negativo. Entretanto, tal compreensão não foi ainda possível [3]. Embora não exista ainda essa compreensão, e por não parecer se tratar de um fato acidental, tentativas de gerar teoricamente a DBN, a partir de princípios gerais de produção de partículas envolvendo interações entre quarks e glúons, foram efetuadas ao longo dos anos. Uma aproximação fenomenológica utilizada para esse propósito é referida como "Clan $\mathrm{Mo}$ del", (Modelo de Clã) introduzida na Física de Altas Energias no XVII International Symposium on Multiparticle Dynamics por L. Van Hove e A. Giovannini [30]. Entretanto, eventual discussão sobre o tema está fora do âmbito deste trabalho. Finalizamos ressaltando que a discussão apresentada neste trabalho, para obtenção da DBN, pode ser motivadora e auxiliar estudos e aplicações a outros sistemas diversos. Há também a possibilidade de ser adaptado para facilitar e viabilizar o ensino de outros modelos de distribuições de probabilidades, como o Binomial e o Geométrico.

\section{Referências}

[1] D. Griffiths, Introduction to Elementary Particles (John Wiley \& Sons, New York, 1987).

[2] W.F. Balthazar e A.L. Oliveira, Partículas elementares no Ensino Médio - Uma Abordagem a partir do LHC (Editora Livraria da Física, São Paulo, 2010).

[3] J.F. Grosse-Oetringhaus e K. Reygers, Journal of Physics G: Nuclear and Particle Physics 37, 083001 (2010).

[4] A. Breakstone, R. Campanini, H.B. Crawley, G.M. Dallavalle, M.M. Deninno, K. Doroba, D. Drijard, F. Fabbri,
M.A. Faessler, A. Firestone et al., Phys. Rev. D 30, 528 (1984).

[5] G.J. Alner, K. Alpgard, P. Anderer, R.E. Ansorge, B. Asman, S. Berglund, K. Berkelman, D. Bertrand, C.N. Booth, C. Buffam et al., Phys. Rep. 154, 247 (1987).

[6] UA 5 Collaboration, R.E. Ansorge, B. Åsman, L. Burow, P. Carlson, R.S. DeWolf, B. Eckart, G. Ekspong, I. Evangelou, C. Fuglesang et al., Z. Phys. C - Particles and Fields 43, 357 (1989).

[7] T. Alexopoulos, E.W. Anderson, N.N. Biswas, A. Bujak, D.D. Carmony, A.R. Erwin, L.J. Gutay, A.S. Hirsch, C. Hojvat, V.P. Kenney et al., Phys. Lett. B 435, 453 (1998).

[8] <http://atlas.physicsmasterclasses.org/en/ zpath_protoncollisions.htm>, acessado em $16 / 05 / 2018$.

[9] P.C. Beggio, M.J. Menon e P. Valin, Phys. Rev. D 61, 034015 (2000).

[10] I.M. Dremin e V.A. Nechitailo, Phys. Rev. D 70, 034005 (2004).

[11] A. Giovannini e R. Ugoccioni, Int. Journal of Modern Phys. A 17, 3897 (2005).

[12] P.C. Beggio e Y. Hama, Braz. J. Phys., 37, 1164 (2007).

[13] P.C. Beggio, Braz. J. Phys., 38, 598 (2008).

[14] P.C. Beggio e E.G.S. Luna, Nucl. Phys. A 929, 230 (2014).

[15] K. Werner e M. Kutschera, Phys. Letters B 220, 243 (1989).

[16] T. Arisawa, Phys. Rev. D 74, 094022 (2006).

[17] A. Giovannini e L. Van Hove, Z. Phys. C 30, 391 (1986).

[18] L.G. Morettin, Estatística Básica - Probabilidade e Inferência (Pearson Education do Brasil, São Paulo, 2009).

[19] P.L. Meyer, Probabilidade - Aplicações À Estatística (Livros Técnicos e Científicos, Rio de Janeiro, 1980).

[20] A.M.L. Freitas e L.C. Laurencel, Variáveis Aleatórias Discretas (Instituto de Matemática e Estatística, Rio de Janeiro, 2008).

[21] M.N. Magalhães, Probabilidade e Variáveis Aleatórias (Editora da Universidade de São Paulo, São Paulo, 2015), $3^{\mathrm{a}}$ ed.

[22] V. Zaccolo, arXiv:1512.05273v1 (2018).

[23] A. Giovannini e R. Ugoccioni, Nuclear Phys. B 64, 68 (1998).

[24] P.C. Beggio, Nucl. Phys. A 864, 140 (2011).

[25] P.C. Beggio, Nucl. Phys. A 913, 264 (2013).

[26] P.C. Beggio, Journal of Phys. G: Nuclear and Particle Phys. 44, 025002 (2017).

[27] M.R. Spiegel, Análise de Fourier (Editora McGraw-Hill do Brasil, São Paulo, 1976).

[28] J.H. Vuolo, Fundamentos da Teoria de Erros (Editora Edgard Blucher, São Paulo, 2005), v. 1, $2^{\text {a }}$ ed.

[29] G.J. Alner, K. Alpgard, P. Anderer, R.E. Ansorge, B. Asman, K. Böckmann, C.N. Booth, L. Burow, P. Carlson, J.L. Chevalley et al., Phys. Lett. B 160, 199 (1985).

[30] L. Van Hove e A. Giovannini, in XVII Symposium on Multiparticle Dynamics, (World Scientific, Singapore, 1987). 